\title{
UN ACERCAMIENTO A LOS DIFERENTES EQUIPOS DE SECADO INDUSTRIAL
}

A RAPPROCHEMENT TO THE DIFFERENT INDUSTRIAL DRYING EQUIPMENT

John Antonny Pabón Beltrán*

https://orcid.org/0000-0002-8534-7497

Luis Eduardo Ayala Padilla**

https://orcid.org/0000-0001-9038-317X

Felipe Correa-Mahecha***

https://orcid.org/0000-0003-1050-8222

FUNDACIÓN UNIVERSIDAD DE AMÉRICA

Recibido: 2 de septiembre de 2018

Aceptado: 24 de junio de 2019

DOI: https://doi.org/10.29097/23461098.305

\section{Resumen}

El proceso de secado ha sido estudiado principalmente en las últimas décadas, ya que se ha logrado estimar que esta técnica puede llegar a consumir hasta un $15 \%$ de toda la energía del proceso de producción. Cada material requiere de unas condiciones de operación específicas, de manera que se han construido equipos de secado que permiten adaptarse a estas condiciones. Este artículo se recopila y analiza información sobre los diferentes equipos de secado industrial y sus principales ventajas y/o avances, así como trata sobre la clasificación y tipos de equipos. La información recopilada fue obtenida de tres bases de datos: SciencieDirect, Knovel y Scopus. El análisis de la información se realizó con la ayuda de las bases de datos mencionadas.

Palabras clave: industria química, secadores, secadores industriales, equipos secado.

\section{Abstract}

The drying process has been reason by study mainly in the last decades since it has been estimated that it can get to consume up to a $15 \%$ of all the energy of the production process, however, as each material requires specific operating conditions, different equipment has been built to simulate these conditions. Throughout this study, information was collected and analyzed on the different industrial drying equipment with its main advantages and / or advances, on the other hand different types of equipment clas-

\footnotetext{
* Ingeniero químico. coinvestigador, grupo de investigación Procesos de separación no convencionales, línea de investigación Procesos de separación sin reacción.

** Ingeniero químico. coinvestigador, grupo de investigación Procesos de separación no convencionales, línea de investigación Procesos de separación sin reacción.

*** Ingeniero químico. Docente investigador, grupo de investigación Procesos de separación no convencionales. $\boldsymbol{\text { felipe.correa@profesores.uamerica.edu.co }}$
} 
sification are mentioned. The information presented in this study was obtained from scientific databases such as SciencieDirect, Knovel and Scopus, finally the studies conducted with the help of the Scopus database were analyzed.

Keywords: chemical industry, dryers, industrial dryers, drying equipment.

\section{INTRODUCCIÓN}

El término "secado" hace referencia a la remoción de cantidades relativamente pequeñas de agua de un material en el caso contrario, si durante el proceso de remoción se retiran grandes cantidades de agua, el proceso se denomina evaporación o destilación (Geankoplis, 1999). El término también es usado frecuentemente para describir procesos en los que se elimina térmicamente la humedad de un producto sólido. El secado suele ser una de las operaciones más complejas de los procesos de fabricación, debido a que es necesario analizar de manera simultánea los fenómenos de transferencia calor, masa y momento (Mujumdar, 2006); además, es un proceso de alto gasto energético, el cual puede llegar a constituir el $15 \%$ de todo el consumo energético industrial (Kudra y Mujumdar, 2009; Kudra, 2007). El secado tiene un área de aplicación muy amplia y es empleado en diferentes industrias, entre las que se encuentran las de alimentos, textiles, farmacéuticos, químicos, entre otros; por esto, además de ser necesario un conocimiento sobre los fenómenos de transporte, es fundamental conocer las características del material para lograr obtener productos de calidad (Strumillo, 2006).Durante la evaporación del solvente la concentración de la solución crece y propiedades como la densidad y la viscosidad aumentan con el contenido de sólidos hasta que la solución se transforma en saturada o resulta inadecuada para una correcta transferencia de calor (García, Peña, López, Duran y Olvera, 2009). Con frecuencia el líquido que hierve se encuentra a un vacío moderado para reducir su temperatura de ebullición, lo que aumenta la diferencia de temperatura entre el vapor condensante y el líquido de ebullición y, por tanto, aumenta la velocidad de transmisión de calor en el evaporador (Seader, Henley y Roper, 2011). Existen diferentes tipos de evaporadores, cada uno con sus respectivas aplicaciones y especificaciones de uso. A continuación, se realiza una revisión bibliográfica sobre los tipos de evaporadores, con el fin de dar al lector una herramienta que permita conocer de primera mano estos equipos.

Durante el proceso de secado, el agua removida de los productos suele variar desde un pequeño rastro hasta una cantidad significativa, como en el caso de las frutas. Es común que la estructura sometida al proceso de secado sufra una reducción de su volumen, del mismo modo, el peso final del producto se reduce; por esto, luego de un proceso de secado se reducen los costos de transporte y se facilita la manipulación del producto final (Cheremisinoff, 2000). Por ejemplo, las hojas sometidas al proceso de secado pueden llegar a perder hasta un $85 \%$ de su peso (Babu, Kumaresan, Raj y Velraj, 2018); los productos biológicos secados o deshidratados prolongan su vida útil debido a la reducción de la actividad del agua, pues el crecimiento microbiano y las reacciones enzimáticas se reducen al mínimo (Vargas y Garzón, 2010). Sin embargo, en el proceso 
de extracción líquida del agua es necesario garantizar que no se produzcan reacciones bioquímicas indeseadas (Babu et ál., 2018).

Los procesos de secado deben ser controlados cuidadosamente debido a la contracción que suelen sufrir los productos, lo que puede ocasionar un deterioro de los mismos; por ejemplo, la estructura del papel se debilita cuando se seca bajo tensión y se evita la contracción lateral, en comparación a lo que ocurre cuando el papel es secado de manera lenta y controlada en un desván (Cheremisinoff, 2000).

Las condiciones en las que se debe llevar a cabo el secado dependen del producto a procesar; en el caso de la leche o las frutas, por ejemplo, el secado debe realizarse rápidamente y a la temperatura más baja posible para que los productos conserven su sabor, olor y otras características valiosas (Cheremisinoff, 2000). Es por esto que la evolución del proceso de secado se ve impulsada por diversos factores, como, por ejemplo: presentación de nuevos enfoques experimentales que permitan la caracterización y control del producto secado; mejoramiento de los equipos tecnológicos existentes; apoyo entre investigadores de distintas disciplinas, entre otros (Tsotsas, Metzger y Peglow 2011).

Como se mencionó anteriormente, el proceso de secado puede ser aplicado en una gran variedad de industrias y cada producto debe ser tratado con unas condiciones de operación especiales para asegurar la calidad final; por lo tanto, la selección del proceso de secado y el diseño del equipo tiene una importancia fundamental en el contenido de humedad, la calidad y el costo del proceso (Kudra y Mujumdar, 2009). Es por esto que la mayoría de los estudios han sido enfocados a dos componentes: la teoría del secado (estudio de los fenómenos de transporte) y equipos de secado (diseño, fabricación u optimización de los equipos, que a su vez están basados en la teoría del secado) (Ryozo, 2007). Aun con el creciente número de investigaciones realizadas al proceso de secado, sigue siendo un reto integrar los conocimientos de termodinámica y fenómenos de transporte en la descripción de equilibrio de fases y la cinética de secado, por lo que este se considera uno de los retos actuales en el proceso (Kowalski, 2000; Kerkhof, 2002).

\section{MÉTODO}

Este documento contextualiza el proceso de secado, trata sobre algunas formas de clasificación de los equipos de secado industrial e identifica varios tipos de estos procesos, para lo cual se recurre a la recolección, procesamiento y análisis de información proveniente de tres bases de datos científicas: SciencieDirec, Knovel y Scopus. La búsqueda de información se realizó en su totalidad en las bases de datos mencionada y se priorizó en investigaciones publicadas entre los años 2000 y 2018 (aunque se analizaron algunos documentos anteriores al 2000). El análisis de la información se realizó con la ayuda de la base de datos bibliográfica Scopus. Durante el proceso de secado, el agua removida de los productos suele variar desde un pequeño rastro hasta una cantidad significativa, como en el caso de las frutas. 


\section{TENDENCIAS CIENTÍFICAS}

El objetivo de este análisis es identificar las tendencias investigativas relativas al proceso de secado. Este estudio se realizó con la base de datos Scopus. Aquí se consideró el período 1978-2018. En este lapso se encuentra el $94.13 \%$ de los documentos publicados con la palabra clave “drying” y se identificaron 39181 investigaciones (la búsqueda se limitó solo a documentos relacionados con la ingeniería). Al realizar la búsqueda no limitada a intervalos de tiempo, pero limitada a documentos relacionados con el campo de la ingeniería, se encontraron 41626 investigaciones.

En la Figura 1 se evidencia un aumento gradual en la producción científica sobre el proceso de secado. El 2017 es el periodo con el mayor número de publicaciones en los últimos cuarenta años, con un total de 2578 documentos, lo que representa el $6.58 \%$ de las publicaciones de las últimas cuatro décadas, seguido por 2014 y 2016, con un total de 2258 (5.76 \%) y 2256 (5.76 \%), respectivamente.

\section{Figura 1}

Número de publicaciones sobre el secado en la base de datos Scopus.

\section{Documents by year}

\section{Scopus}

3000

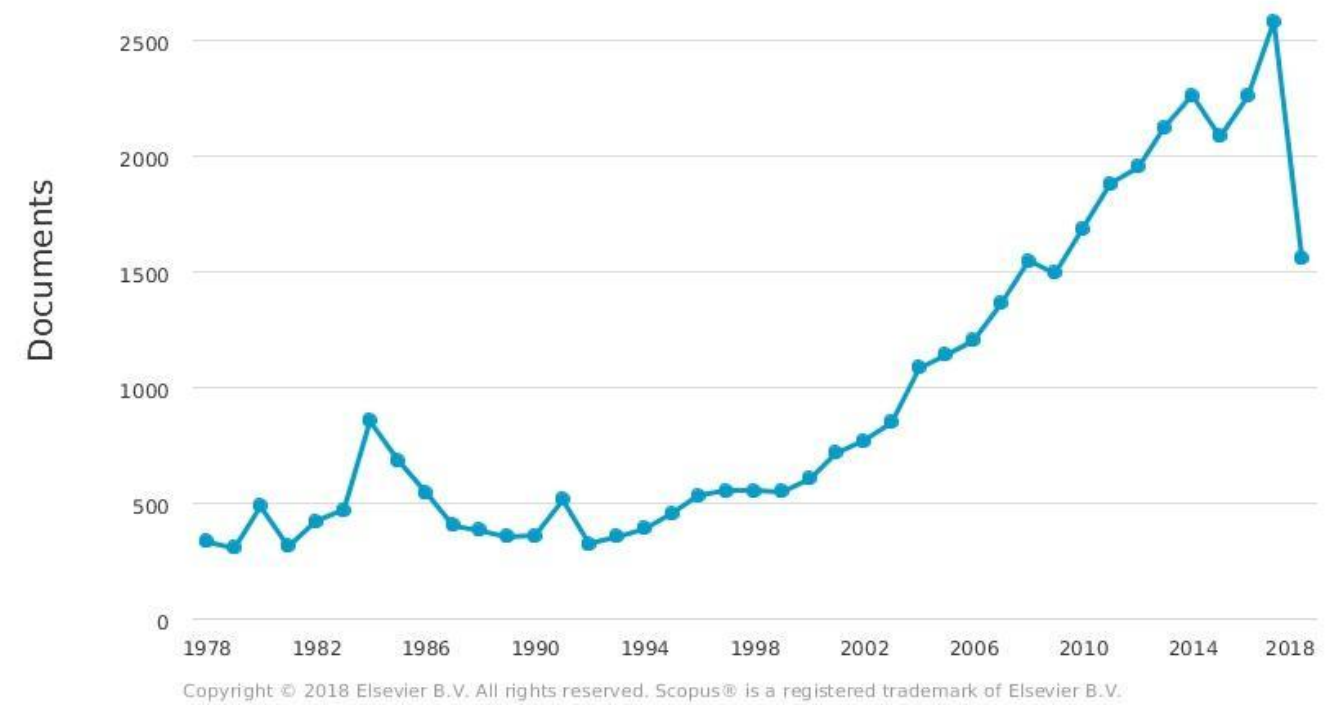

Fuente: Scopus (2018).

En la Figura 2 se representan las publicaciones con la palabra clave “drying”. Se publicaron un total de 27331 artículos, que corresponden al $69.8 \%$ del total de los documentos publicados sobre el tema, seguido de conferencias, que representan el 24.6 $\%$, y revisiones, que equivalen al 1.9\%, con 763 documentos (el porcentaje restante corresponde a otro tipo de publicaciones, libros, reportes, notas, entre otros). 
Figura 2

Distribución de las publicaciones de acuerdo con el tipo de documento.

Documents by type

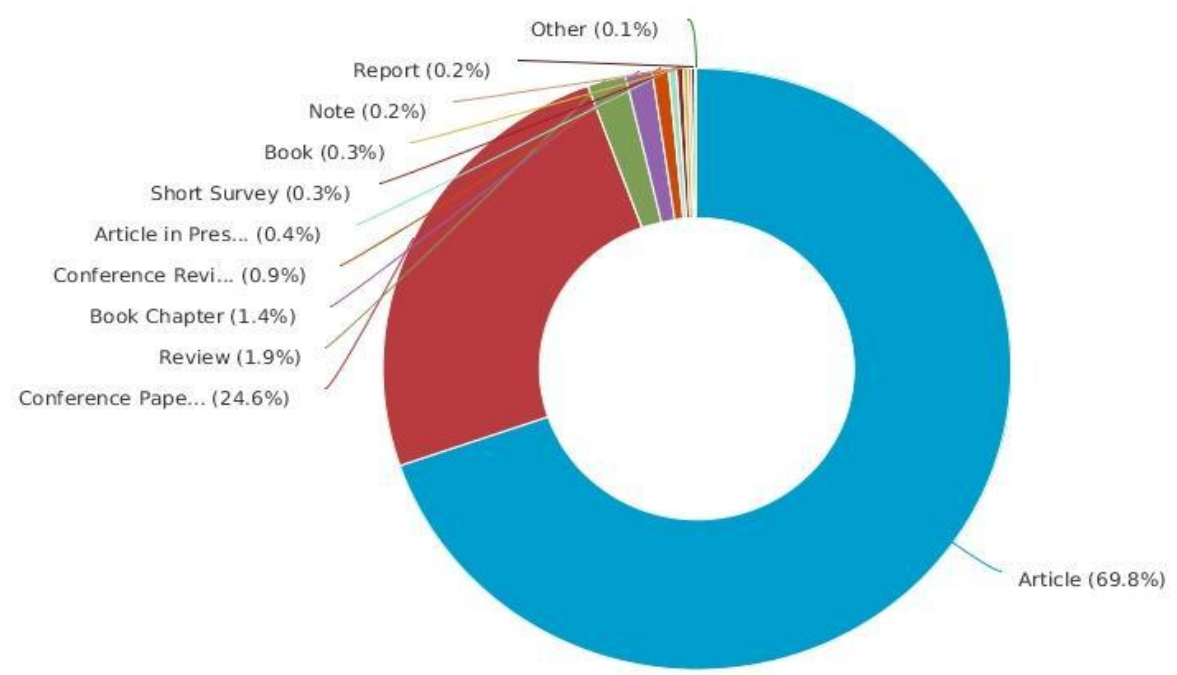

Copyright 2018 Elsevier B.V. All rights reserved. Scopusio is a registered trademark of Elsevier B.V.

Fuente: Scopus (2018).

\section{CLASIFICACIÓN DE LOS EQUIPOS DE SECADO}

Los equipos empleados para el proceso de secado de los diferentes materiales han sido clasificados y diversificados de acuerdo con las especificaciones y requisitos de cada producto (Yayaganbaba y Kurtbaş, 2016). Existen diferentes formas de clasificar este tipo de equipos, entre las que se encuentran: modo de operación, tipo de calor de entrada (o método de transferencia de calor), presión operacional, tiempo de reincidencia, entre otros (Kudra y Mujumdar, 2009). A lo largo de este artículo se identifican distintos equipos de secado, que se categorizan según su clasificación por el método de transferencia de calor.

Según este tipo de clasificación, los equipos de secado se dividen en tres grupos: directos, indirectos (Ocon y Tojo, 1974) y diversos (Universidad Nacional Francisco de Miranda, s. f.); no obstante, algunos autores dividen este último grupo en equipos de radiación y de calentamiento dieléctrico (Ocon y Tojo, 1974).

\section{Equipos directos}

En este tipo de equipos el calor necesario para realizar el proceso de secado es suministrado directamente por aire caliente, el cual entra en contacto directo con el 
material, por lo que también se les conoce como secadores convectivos (Ocon y Tojo, 1974).

Los secadores de pulverización o aspersión emplean un aspersor o espray con el fin de adquirir un producto seco en forma de polvo (Mujumdar, 2006). El equipo consiste principalmente en una cámara cerrada en donde se atomizada una suspensión, la cual tiene disuelto el sólido que se desea secar. La corriente de aire empleada en estos equipos puede llegar a alcanzar una temperatura de $300{ }^{\circ} \mathrm{F}$ y el sólido tarda aproximadamente entre 15 y 30 segundos en completar su proceso de secado (Cheremisinoff, 2000).

\section{Figura 3}

Equipo de secado por aspersión o pulverización.

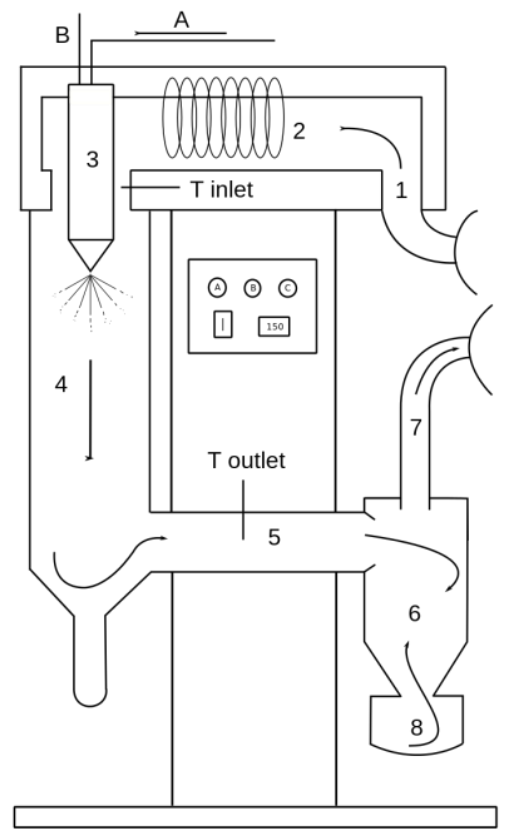

Nota A = solución o suspensión a secar; $\mathrm{B}$ = gas de atomización; 1 = gas de secado; 2 = calentamiento del gas de secado; 3 = rociado de solución o suspensión; 4 = cámara de secado; 5 = parte entre la cámara de secado y el ciclón; 6 = ciclón; 7 = gas de secado seco; 8 = recipiente de recolección del producto. Fuente: Pelkonen (2009).

Los secadores rotatorios constan esencialmente de un cilindro horizontal ligeramente inclinado, que puede ser rotatorio o tener un agitador interno, el cual moverá el material que se desea secar (Cheremisinoff, 2000). Algunas de las principales características de los secadores rotatorios son: secado eficiente de materiales, tamaño del equipo variable, alta eficiencia térmica, entre otras. Por otro lado, estos equipos no son adecuados para bajas tasas de producción; además, su mantenimiento varía de acuerdo con el material 
que será secado (Lisboa, Vitorino, Delaiba, Finzer y Barrozo, 2007; Renaud, Thaibault y Tursiak 2000).

Figura 4

\section{Secador rotatorio}

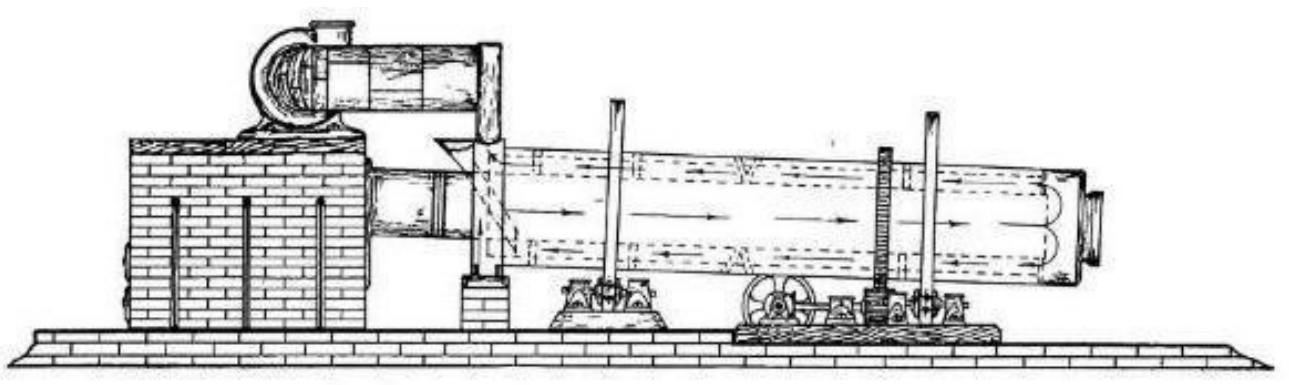

Fuente: Kreitzer (2012).

Los secadores de cinta transportadora son equipos de secado que tienen una serie de compartimientos de bandejas en las que se depositan los sólidos, las que durante su recorrido atraviesan una cámara en la que circulan gases calientes (Geankoplis, 1999). Los compartimientos usualmente se encuentran sobre una cinta transportadora o dos cadenas sin fin en forma de cinturón. Este proceso puede tardar varios minutos y a la salida de la cámara normalmente un operario descarga el contenido de las bandejas que completaron el recorrido y deposita nuevo material. El proceso se realiza de manera lenta, de modo que se necesite la menor cantidad posible de mano de obra (Cheremisinoff, 2000). Generalmente, el secador de cinta transportadora es la mejor opción para el secado de material en partículas, con un intervalo de 1 a $50 \mathrm{~mm}$ de diámetro, además de ser uno de los equipos más disponibles en la industria (Yayaganbaba y Kurtbaş, 2016).

Los secadores de lecho fluidizado son adecuados para una amplia gama de procesos, especialmente para polvos, gránulos, aglomerados y gránulos con un tamaño medio de partícula de entre 50 micras y 5 mm (Yayaganbaba y Kurtbaş, 2016). La principal característica de este tipo de equipos es el flujo tangencial entre el sólido que se desea secar y la corriente de aire caliente (Treybal, 1988). Gracias a la amplia gama de procesos que pueden llevarse a cabo con este tipo de equipos en las últimas décadas, se ha evidenciado un creciente número de investigaciones sobre estos; algunas muestran los avances recientes, como es el caso de la investigación de Daud (2008); sin embargo, otras se han enfocado principalmente en el proceso de modelación y creación de modelos matemáticos, los cuales permiten conocer más sobre el funcionamiento de estos equipos (véase Garvavi, Kasiri, Hashemadabi, 2006; Panda y Ramachandra, 1991)

Los secadores neumáticos siguen el mismo principio que los secadores de lecho fluidizado, es decir, la corriente de aire golpea tangencialmente a las partículas sólidas; no obstante, estos equipos aumentan la velocidad del aire hasta la velocidad terminal de 
las partículas sólidas individuales, lo que produce que estas viajen en el mismo sentido que la corriente de aire una vez se encuentran con ella. El tiempo de secado en estos equipos es de tan solo unos segundos, por lo que el proceso se ve limitado a casos en los que solo se tiene humedad superficial (Cheremisinoff, 2000).

Los secadores por liofilización se emplean para secar materiales muy sensibles al calor. Estos equipos son usados principalmente por la industria farmacéutica (Liapis, Pim y Bruttini, 1996). Durante el proceso de liofilización el material a secar generalmente se congela exponiendolo al aire a bajas temperaturas; por su parte, la humedad es retirada como vapor por sublimación. Este es uno de los procesos de secado más costosos, debido a que es necesario emplear vacío y porque es un método lento (Geankoplis, 1999).

\section{Equipos indirectos}

En los secadores indirectos el calor se transfiere de una fuente térmica a través de una placa (generalmente metálica), la cual entra en contacto con el material a secar (TOJO, 1974). En estos equipos la placa metálica encargada de suministrar el calor necesario para realizar el proceso se calienta con la ayuda de un fluido caliente que viaja alrededor de ella. El fenómeno de transferencia de calor dominante se da por conducción (Lattman y Laible, 2005).

Los secadores de tambor rotatorio son de forma cilíndrica y han comenzado a ser empleados en la producción de una gran cantidad de productos, desde comida para bebés hasta químicos, esto gracias a su capacidad de secar productos altamente pegajosos y/o medios altamente viscosos (Yayaganbaba y Kurtbaş, 2016). Los materiales que se secan en estos equipos se vierten sobre la superficie interna del tambor, que se calienta y gira proporcionando el calor necesario para retirar la humedad; el tambor suele rotar lentamente, de modo que luego de siete octavos de revolución ya es posible retirar un depósito seco, el que generalmente se extrae por medio de un cuchillo flexible que rueda hacia un contenedor o un transportador de tornillo. Además, tiene distintas distribuciones en las que se puede emplear doble tambor con alimentación central o por salpicadura (Cheremisinoff, 2000).

Los secadores de anaqueles al vacío son similares a los secadores de bandejas, con la excepción de que en estos sus gabinetes y bandejas se encuentran construidos en acero o hierro colado; aparte de esto, tienen puertas herméticas, lo que les permite operar a presiones inferiores a la atmosférica (Treybal, 1988). En estos equipos el calor necesario para realizar el proceso es suministrado por medio de agua tibia (o vapor), la cual circula por los anaqueles huecos (Geankoplis, 1999).

Secadores giratorios de vacío son empleados normalmente para secar lodos o pastas de gran valor. Este secador consta de cubiertas cilíndricas con chaqueta de vapor, la que suministra el calor necesario para la evaporación de la humedad. El lodo es agitado por hojas, mientras la humedad retirada pasa por un condensador; la humedad que no se puede condensar es retirada con la ayuda de una bomba de vacío (Treybal, 1988). 


\section{Equipos de secado por radiación}

Estos equipos suministran el calor necesario para llevar a cabo el proceso por medio de fuentes de radiación; generalmente emplean radiación solar o radiación infrarroja (Suárez, Barrera y Forero, 2016).

El secador de infrarrojos se ha vuelto popular en los últimos años. Si bien el primer artículo acerca de este tipo de equipos fue publicado en 1952 (véase Broughton y Gilman, 1952), desde inicios del 2000 se ha visto un importante incremento de investigaciones publicadas sobre el tema. El método de transferencia de calor por radiación infrarroja proporciona algunas ventajas significativas con respecto a los procesos de secado comunes; algunas de estas ventajas son ahorro de tiempo, ahorro de energía, alta calidad del producto, entre otras (Yayaganbaba y Kurtbaş, 2016). Aparte de esto, se han realizado diferentes investigaciones sobre modelos matemáticos que permiten un control avanzado del proceso (Dhib, 2007), e incluso estudios que se enfocan en la simulación del proceso con este tipo de equipos (Rajan, Irudayaraj y Jun, 2002).

En los secadores solares el material está dispuesto sobre bandejas (generalmente de acero inoxidable) y es expuesto directamente a radiación solar, que proporciona el calor necesario para realizar el proceso (Suárez et ál., 2016). Algunas ventajas de este tipo de secadores son sus bajos costos de operación (en comparación a los medios mecánicos) y de capital y poca experiencia para su uso; por otra parte, sus desventajas son riesgo de contaminación, robo o daño por plagas, secado intermitente, posible formación de moho, calidad variable de los productos, entre otras (Sabbagh, 1977). Entre otros tipos de secadores solares, estos se clasifican por el modo de circulación del aire: natural o forzada (Yayaganbaba y Kurtbaş, 2016). Igualmente, se han realizado diversos estudios sobre el rendimiento de los diferentes secadores solares (Mustayen, 2014), las aplicaciones en las que han sido empleados (Pirasteha, Saidurab, Rahmanc y Rahimb 2014) e incluso los sistemas de secado solar (Sharma, Chen y Lan, 2009).

\section{Figura 5}

Secador solar directo.

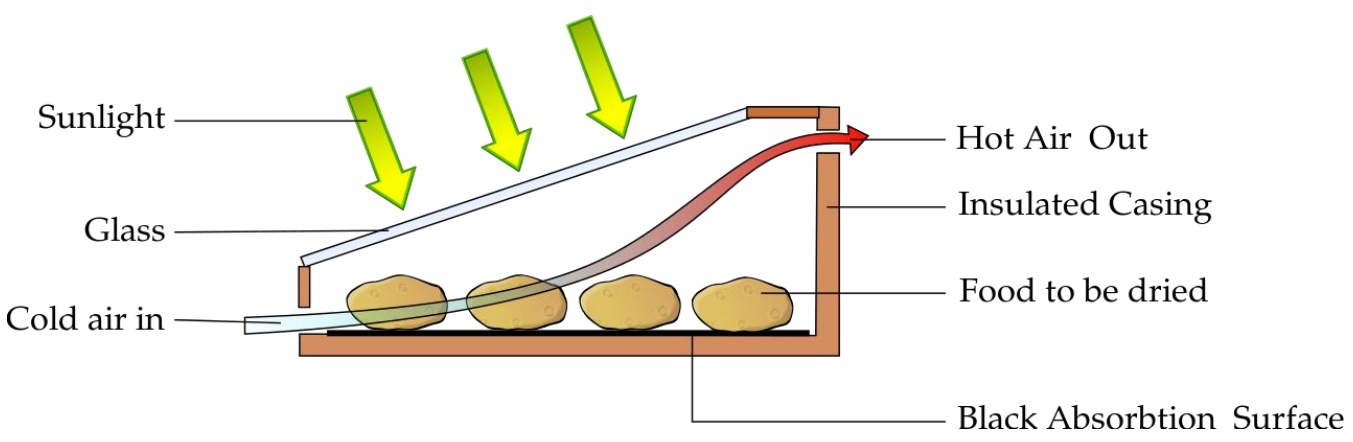

Fuente: Karthikeyan (2013). 


\section{Equipos de secado por calentamiento dieléctrico}

El calentamiento dieléctrico es el proceso de secado por el cual mediante ondas de radio, microondas o radiación electromagnética se calienta un material dieléctrico. La ventaja de este tipo de calentamiento es que se produce directamente dentro del material expuesto (por lo que su calentamiento es más uniforme) y consume generalmente menos energía; así, por ejemplo, en el calentamiento por radiofrecuencia la frecuencia aplicada se encuentra entre $1.3 \times 10^{7}$ y $2.45 \times 10^{9} \mathrm{~Hz}$ (Hertz), mientras que en el calentamiento con microondas la frecuencia está entre $9.15 \times 10^{8}$ y 2.45 x $10^{9} \mathrm{~Hz}$ (Yayaganbaba y Kurtbaş, 2016). La frecuencia empleada por estos equipos es incluso menor a la frecuencia del espectro visible, la que se encuentra entre 3.84 x $10^{14}$ y 7.89 x $10^{14} \mathrm{~Hz}$. Sin embargo, también se ha logrado identificar que los rangos de frecuencia empleados son más amplios que el rango del espectro visible (Hernández, 2013). Por otra parte, la primera investigación de este tipo de proceso de secado se desarrolló en 1949 (véase Mann, Ceaglske y Olson, 1949) y solo en las últimas décadas ha cobrado importancia.

A medida que se han conocido las propiedades y ventajas de este tipo de calentamiento se ha generado un mayor interés en la comunidad científica, por lo que se han realizado estudios en los que se describe el funcionamiento y los desarrollos de este tipo de equipos (Jones, 1989), incluso se han realizado investigaciones enfocadas al secado dieléctrico (véase Jones y Rowley, 1996).

\section{CONCLUSIONES}

Este artículo revisa diferentes investigaciones sobre los equipos de secado diseñados para la industria. Aquí se describe las características y modo de operación de los equipos, explicando el motivo por el cual son diseñados de diferentes maneras. Así, cada diseño tiene el objetivo de mejorar el proceso para determinados tipos de material, lo que puede favorecer tanto la calidad de los productos sometidos a este proceso, como el costo del proceso en sí mismo.

Es necesario considerar las características de los materiales que se someten a los procesos de secado y realizar una revisión de los equipos más adecuados para llevar a cabo el proceso, ya que de no tener en cuenta esta información es posible adquirir un equipo insuficiente y/o no apropiado para el proceso, lo que generara un mayor gasto económico en la industria, aún más si se tiene en cuenta que el proceso de secado es un método de alto consumo energético.

\section{Referencias}

Babu, A., Kumaresan, G., Raj, V., y Velraj, R. (2018). Review of leaf drying mechanism and inflencing parameters, drying method, nutrient preservation, and mathematical models. Renewable and Sustainable Energy Reviews, 90, 536556. 
Broughton, G., y Gilman, L. (1952). A flexible laboratory infrared heater-dryer. Journal of Chemical Education, 29(1), 34.

Cheremisinoff, P. (2000). Handbook of chemical processing equipment. Elsevier.

Daud, W. (2008). Fluidized bed dryers-Recent advances. Advanced Powder Technology, 19(5), 403-418.

Dhib, R. (2007). Infrared drying: from process modeling to advanced process control. Drying Technology, 25(1), 97-105.

Garvavi, L., Kasiri, N., y Hashemadabi, S. (2006). Mathematical modeling of a continuous fludized bed dryer. International Communications in Heat and Mass Transfer, 33(5), 666-675.

Geankoplis, C. (1999). Procesos de transporte y operaciones unitarias ( $3^{\mathrm{a}}$ ed.). CECSA.

Jones, P. (1989). Dielectric-assisted drying and processing. Power Engineering Journal, 3(2), 59-66.

Jones., P., y Rowley, A. (1996). Dielectric drying. Drying technology, 14(5), 10631098.

Karthikeyan, A. (2013). Direct solar dryder [imagen original en formato JPG.]. https://commons.wikimedia.org/wiki/File:Direct_Solar_dryder.svg

Kerkhof, P, y Coumans, W. (2002). Drying: a fascinating unit operation. Chemical Engineering Journal, 1(86), 1-2.

Kowalski, S, (2000). Toward a thermodynamics and mechanics of drying processes. Chemical Engineering Science, 55(7), 1289-1304.

Kudra, T. (200747) Energy aspects in drying. An International Journal, 22(5), 917-932. Kudra, T., y Mujumdar, A. (2009). Advanced drying technologies (2a ed.). CR Press.

Kreitzer, H. (1924). Rotary dryer [imagen original en formato JPG]. https://commons.wikimedia.org/wiki/File:Rotary_dryer.JPG

Lattman, M., y Laible, R. (2005). Batch drying: the indirect solution to sensitive frying problems. Chemical Engineering, 112(12), 34-40.

Liapis, M., Pim, M., y R. Bruttini, R. (1996). Research and development needs and opportunities in freeze dryingfreeze-drying. Drying Technology, 14(6), 12651300.

Lisboa, M., Vitorino, D., Delaiba, W., Finzer, J., y Barrozo, M. (2007). A study of particle motion in rotary dryer. Available. Brazilian Journal of Chemical Engineering, 24(3), 365-374.

Mann, C., Ceaglske, H., y Olson, A. (1949). Mechanism of dielectric drying. Industrial and Engineering Chemistry, 41(8), 1686-1694.

Mujumdar, A. (Ed.). (2006) Handbook of industrial drying (3a ed.). Taylor \& Francis.

Mustayen, A., Mekhilef, S., y Saidur, R. (2014). Performance study of different solar dryers: a review. Renewable and Sustainable Energy Reviews, 34, 463-470.

Ocon, J., y Tojo, G. (1974). Problemas de ingeniería química (vol. II). Aguilar.

Panda, R., y Ramachandra V. (1991). Fluidized bed dryers: dynamic modelling and control. Chemical Engineering \& Technology: Industrial Chemistry-Plant Equipment-Process Engineering-Biotechnology, 14(5), 307-310

Pelkonen, T. (2009). Labspraydryer [imagen original en formato PNG]. https://es.m.wikipedia.org/wiki/Archivo:Labspraydryer.svg 
Pirasteha, G., Saidurab, R., Rahmanc, S., y Rahimb, N. (2014) A review on development of solar drying applications. Renewable and Sustainable Energy Reviews, 31, 133-148.

Rajan, R., Irudayaraj, J., y Jun, S. (2002). Simulation of infrared drying process. Drying Technology, 20(2), 363-379.

Renaud, M., Thaibault, J., y Tursiak, A. (2000). Solids transportation model of an industrial rotary dryer. Drying Technology, 18(4-5), 843-865.

Ryozo, T. (2007) Theoretical fundamentals of drying operation. Drying Technology, 14(1), 1-7.

Sabbagh, J. (1977). Heat transfer for solar energy utilization. En A. Sayigh, Solar energy engineering (pp. 83-103). Academic Press.

Scopus (2018). Analyze search results, Document by year. https://ezproxy.uamerica. edu.co:2080/term/analyzer.uri?sid=73124db2ae7af043e33a46f6222776a5\&o rigin=resultslist\&src=s\&s=TITLE-ABS-KEY\%28drying\%29\&sort=plf$\underline{\mathrm{f} \& \mathrm{sdt}=\mathrm{b} \& \mathrm{sot}=\mathrm{b} \& \mathrm{sl}=21 \& \mathrm{count}=191500 \& a n a l y z e R e s u l t s=\text { Analyze}+ \text { results \&t }}$ xGid=cda9946827bdb048d82c91ead9fc3024

Scopus (2018). Analyze search results, Document by type. https://ezproxy.uamerica. edu.co:2080/term/analyzer.uri?sid=73124db2ae7af043e33a46f6222776a5\&o rigin=resultslist\&src=s\&s=TITLE-ABS-KEY\%28drying\%29\&sort=plf-

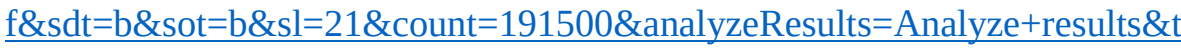
xGid=cda9946827bdb048d82c91ead9fc3024

Sharma, A., Chen, C., y vu Lan, N. (2009). Solar-energy drying systems: a review. Renewable and sustainable energy reviews, 13(6-7), 1185-1210.

Strumillo, C. (2006). Perspectives on the evolution of drying. Drying Technology Technol Seca, 24(9), 1059 -10 68.

Suárez, L., Barrera, R., y Forero Sandoval, A. (2016). Evaluación de alternativas de secado en el proceso de elaboración de harina de lombriz. Revista Corpoica: Ciencia y Tecnología Agropecuaria, 17(1), 55-71.

Treybal, R. (1988). Operaciones de transferencia de masa (2 $2^{\mathrm{a}}$ ed.). McGraw-Hill.

Tsotsas, E., Metzger, T., y Peglow, M. (2011). New developments in drying. Chemical, Engineering and Technology, 34(7), 1023.

Vargas, E., y Garzón, F. (2010). Designing, assembly and start up for a semiautomatic hot-air tray dryer. Ingeniería e Investigación, 30(2), 43-51.

Yayaganbaba, A., y Kurta, İ (2016). A scientific approach with bibliometric analysis related to brick and tile drying: a review. Renewable and Sustainable Energy Reviews, 59, 206-224. 